\title{
cGMP-dependent signaling pathways in spinal pain processing
} Achim Schmidtko*1, Wei Gao ${ }^{1}$, Peter König' ${ }^{2}$, Sandra Heine ${ }^{1}$, Matthias Sausbier ${ }^{3}$, Peter Ruth ${ }^{3}$, Doris Koesling ${ }^{4}$, Irmgard Tegeder ${ }^{1}$, Andreas Friebe ${ }^{4}$ and Gerd Geisslinger ${ }^{1}$

\author{
Address: ${ }^{1}$ Pharmazentrum Frankfurt/ZAFES, Institut für Klinische Pharmakologie, Goethe-Universität, Frankfurt am Main, Germany, ${ }^{2}$ Institut für \\ Anatomie, Universität Lübeck, Germany, ${ }^{3}$ Pharmazeutisches Institut, Pharmakologie und Toxikologie, Universität Tübingen, Germany and \\ ${ }^{4}$ Institut für Pharmakologie und Toxikologie, Ruhr-Universität, Bochum, Germany \\ Email: Achim Schmidtko* - schmidtko@em.uni-frankfurt.de \\ * Corresponding author
}

from 4th International Conference of cGMP Generators, Effectors and Therapeutic Implications

Regensburg, Germany. 19-21 June 2009

Published: II August 2009

BMC Pharmacology 2009, 9(SuppI I):S37 doi:I0.II86/I47I-22I0-9-SI-S37

This abstract is available from: http://www.biomedcentral.com/I47I-22/0/9/SI/S37

(c) 2009 Schmidtko et al; licensee BioMed Central Ltd.

\section{Background}

An exaggerated pain sensitivity is the dominant feature of inflammatory and neuropathic pain both in the clinical setting and in experimental animal models. It manifests as pain in response to normally innocuous stimuli (allodynia), increased response to noxious stimuli (hyperalgesia) or spontaneous pain, and can persist long after the initial injury is resolved. Research over the last decades has revealed that several signaling pathways in the spinal cord essentially contribute to the pain sensitization. To test the contribution of cGMP produced by NO-sensitive guanylyl cyclase (NO-GC) to pain sensitization, we investigated the localization of NO-GC in the spinal cord and in dorsal root ganglia, and we characterized the nociceptive behavior of mice deficient in NO-GC (GC-KO mice).

\section{Results}

We show that NO-GC ( $\beta_{1}$ subunit) is distinctly expressed in neurons of the mouse spinal cord, while its distribution in dorsal root ganglia is restricted to non-neuronal cells. GC-KO mice exhibited a considerably reduced nociceptive behavior in models of inflammatory or neuropathic pain, but their responses to acute pain were not impaired. Moreover, GC-KO mice failed to develop pain sensitization induced by spinal administration of drugs releasing NO. Surprisingly, during spinal nociceptive processing cGMP produced by NO-GC may activate signaling path- ways different from cGMP-dependent protein kinase I (cGKI), while cGKI can be activated by natriuretic peptide receptor-B (NPR-B) dependent cGMP production.

\section{Conclusion}

Taken together, our results provide evidence that NO-GC has a dominant role in the development of exaggerated pain sensitivity during inflammatory and neuropathic pain. Furthermore, beside the NO-mediated cGMP synthesis, cGMP produced by NPR-B contributes to pain sensitization by activation of CGKI.

\section{Acknowledgements}

Supported by the Deutsche Forschungsgemeinschaft (DFG-SFB 8I5/AI4). 